\title{
Downregulation of IncRNA BANCR participates in the development of retinopathy among diabetic patients
}

\author{
XIAOXIA ZHANG ${ }^{1}$, XINRONG ZOU ${ }^{2}$, YUTING LI $^{1}$ and YUPING WANG ${ }^{1}$ \\ ${ }^{1}$ Department of Ophthalmology, Lanzhou University Second Hospital, Lanzhou, Gansu 730030; \\ ${ }^{2}$ Department of Ophthalmology, Shanghai Fengcheng Hospital, Shanghai 200000, P.R. China
}

Received September 3, 2018; Accepted February 22, 2019

DOI: $10.3892 /$ etm.2019.7444

\begin{abstract}
The long non-coding (lnc)RNA B-Raf protooncogene, serine/threonine kinase-activated non-protein coding RNA (BANCR) is a well-characterized oncogene, while its potential functions in other diseases remain elusive. In the present study, the possible association of BANCR with diabetic retinopathy was investigated. A total of 244 patients with diabetes were followed up every 6 months for 8 years to record the occurrence of retinopathy. A total of 38 patients developed diabetic retinopathy. During the follow-up, the plasma levels of lncRNA BANCR decreased in those patients with diabetic retinopathy but not in those with other complications or without any complications. The plasma levels of lncRNA BANCR at 12 months prior to the diagnosis of diabetic retinopathy are able to sufficiently distinguish diabetic retinopathy patients from healthy controls and diabetic patients without any obvious complications. In vitro, high-glucose treatment failed to affect the expression of lncRNA BANCR in the human retinal pigment epithelial cell line ARPE-19. However, lncRNA BANCR overexpression inhibited the apoptosis of ARPE-19 cells under high-glucose conditions. Therefore, it is indicated that IncRNA BANCR participates in the development of retinopathy in diabetic patients through its regulatory role in cell apoptosis, and may serve as a novel prognostic indicator and therapeutic target.
\end{abstract}

\section{Introduction}

Diabetic retinopathy, also referred to as diabetic eye disease, is a type of damage to the retina caused by the high-glucose environment in patients with diabetes mellitus (1). Clinical studies have indicated that diabetic retinopathy is a leading cause of blindness in different populations all over the world (2). An

Correspondence to: Dr Yuping Wang, Department of Ophthalmology, Lanzhou University Second Hospital, 82 Cuiyingmen, Chengguan, Lanzhou, Gansu 730030, P.R. China E-mail: ovqhn41@163.com

Key words: diabetic retinopathy, long non-coding RNA BANCR, retinal pigment epithelial cell, apoptosis estimated $10-50 \%$ of patients with diabetes eventually develop diabetic retinopathy during the course of the disease (3). With the increasing incidence rate of diabetes mellitus $(4,5)$, the prevalence of diabetic retinopathy is expected to further increase in the near future. In spite of efforts to improve the treatment strategies for diabetic retinopathy, blindness inevitably occurs in certain cases $(6,7)$. However, the underlying mechanisms remain largely elusive. At present, early detection followed by active treatment remains key in the management of diabetic retinopathy.

Long non-coding RNAs (lncRNAs) is a group of non-coding RNAs comprising $>200$ nucleotides. A growing body of evidence has revealed that certain IncRNAs are key factors in various human diseases $(8,9)$. However, the involvement of IncRNAs in diabetic retinopathy has remained largely unexplored. LncRNA B-Raf proto-oncogene, serine/threonine kinase-activated non-protein coding RNA (BANCR) is a well-characterized lncRNA in human cancers $(10,11)$. Of note, IncRNA BANCR was recently proved to participate in retinoblastoma, indicating its potential involvement in other eye diseases (12). In the present study, it was demonstrated that IncRNA BANCR participated in the development of retinopathy among diabetic patients, possibly through its regulatory role in cell apoptosis.

\section{Materials and methods}

Cell line and patients. The human retinal pigment epithelial (RPE) cell line ARPE-19 was purchased from the American Type Culture Collection (ATCC; Manassas, VA, USA). ARPE-19 cells were with normal karyology and form polarized epithelial monolayers on porous filter supports. ARPE-19 cells have structural and functional properties resembling those of RPE cells in vivo (13). Cells were cultivated with ATCC-formulated Dulbecco's modified Eagle's medium (DMEM)/F12 (30-2006 ${ }^{\mathrm{TM}}$; ATCC) containing 10\% fetal bovine serum in an incubator $\left(37^{\circ} \mathrm{C}, 5 \% \mathrm{CO}_{2}\right)$.

A total of 244 patients with type II diabetes were enrolled in the present study. Those patients were diagnosed and treated at Lanzhou University Second Hospital (Lanzhou, China) between March 2008 to March 2010 and subsequently treated. The following diagnostic criteria were used: i) Random plasma glucose $\geq 11.1 \mathrm{mmol} / 1$ with diabetic symptoms (polyuria, fatigue, polydipsia or weight loss); and ii) 2-h post-load glucose 
$\geq 11.1 \mathrm{mmol} / \mathrm{l}$ following $75 \mathrm{~g}$ oral glucose uptake. The inclusion criteria were as follows: i) Patients diagnosed with diabetes for the first time; ii) patients without any obvious diabetic complications; iii) patients completed 8-year follow-up; iv) patients developed a single diabetic complication. The exclusion criteria were as follows: i) Patients complicated with multiple diseases; ii) patients had already developed diabetic complications at the time-point of diagnosis; iii) patients who were treated within 90 days prior to admission; iv) patients died during follow-up; v) patients developed multiple diabetic complications. At the same time, the present study also included 102 healthy volunteers to serve as a control group. All healthy volunteers exhibited all physiological parameters within normal range following systemic physiological examinations. The patient group included 133 males and 111 females, and the age ranged from 30 to 67 years, with an average age of $46.4 \pm 5.5$ years. The control group included 60 males and 52 females, and the age ranged from 28 to 69 years, with an average age of $48.1 \pm 6.2$ years. The patient and control groups had similar age and gender distributions. The present study passed the review of the Ethics Committee of Lanzhou University Second Hospital (Lanzhou, China). All participants provided written informed consent.

Follow-up and plasma samples. Blood was extracted from each participant on the day of admission. Patients were followed-up for 8 years to record the occurrence of any diabetic complications. Patients were followed up every 6 months and blood was extracted on each visit. Plasma was prepared using conventional methods. All specimens were stored in liquid nitrogen prior to analysis.

Reverse transcription-quantitative polymerase chain reaction $(R T-q P C R)$. RNAzol ${ }^{\circledR}$ RT RNA Isolation Reagent (Genecopoeia, Guangzhou, China) was used to extract total RNA. After RT using the RevertAid RT Reverse Transcription kit (cat. no. K1691; Thermo Fisher Scientific, Inc., Waltham, MA, USA), PCR mixtures were prepared using the SensiFAST ${ }^{\text {тм }}$ Real-Time PCR kit (cat. no. BIO-86005; Bioline, London, UK). The reaction conditions were $45 \mathrm{sec}$ at $95^{\circ} \mathrm{C}$, followed by 40 cycles of $28 \mathrm{sec}$ at $95^{\circ} \mathrm{C}$ and $36 \mathrm{sec}$ at $56^{\circ} \mathrm{C}$. Sequences of primers used for PCR were as follows: IncRNA BANCR forward, 5'-ACAGGACTCCATGGC AAACG-3' and reverse, 5'-ATGAAGAAAGCCTGGTGC AGT-3'; $\beta$-actin forward, 5'-GACCTCTATGCCAACACA GT-3' and reverse, 5'-AGTACTTGCGCTCAGGAGGA-3'. Quantitative expression values were determined using the $2^{-\Delta \Delta \mathrm{Cq}}$ method (14).

Cell transfection. pIRES2 vectors expressing lncRNA BANCR and empty pIRES2 vectors were purchased from Genecopoeia. LncRNA BANCR small interfering (si)RNA and negative control siRNA were designed and synthesized by Sangon Biotech Co., Ltd. (Shanghai, China). Transfection was performed using Lipofectamine $2000^{\mathrm{TM}}$ reagent (Invitrogen; Thermo Fisher Scientific, Inc.) with the respective vectors at a dose of $15 \mathrm{nM}$. Untransfected cells were used as control cells and cells transfected with empty vector were used as negative control cells. The cells were subjected to the respective assays at $24 \mathrm{~h}$ after transfection.
Cell apoptosis assay. Cells with an IncRNA BANCR overexpression rate of $>200 \%$ and their controls were subjected to the apoptosis assay. In brief, cell suspensions $\left(6 \times 10^{4}\right.$ cells $\left./ \mathrm{ml}\right)$ were prepared using serum-free medium. Cells were cultivated in a 6-well plate with $10 \mathrm{ml}$ cell suspension in each well, followed by the addition of 5, 10, 20 or $40 \mathrm{mM}$ d-glucose. After the cells were cultivated for $48 \mathrm{~h}$ (15), digestion with $0.25 \%$ trypsin was performed, and the cells were collected and suspended in DMEM medium. subsequently, Annexin V-fluorescein isothiocyanate (FITC; Dojindo, Kumamoto, Japan) and propidium iodide (PI) staining was performed and apoptotic cells were detected by flow cytometry using WOLF Cell Sorter (NanoCellect Biomedical, Inc., San Diego, CA, USA).

Statistical analysis. All experiments were performed in triplicate and values are expressed as the mean \pm standard deviation. The diagnostic value of the plasma levels of lncRNA BANCR for diabetic retinopathy was evaluated by receiver operating characteristic (ROC) curve analysis. Student's t-test was used for comparisons between two groups. One-way analysis of variance followed by Tukey's test was used for comparisons among multiple groups. $\mathrm{P}<0.05$ was considered to indicate statistical significance.

\section{Results}

Occurrence of diabetic complications during 8-year follow-up. During the 8-year follow-up, diabetic retinopathy occurred in 38 cases (occurred 18-96 months following admission; median, $60.7 \pm 22.3$ months), diabetic cardiomyopathy in 23 cases, diabetic neuropathy in 56 cases and other complications in 28 cases. No obvious complications were detected in the 99 remaining cases. The basic information of the patients on the day of admission is provided in Table I. No significant differences in the baseline data were detected among patients with different complications.

LncRNA BANCR was not differentially expressed in diabetic patients and healthy controls on the day of admission. RT-qPCR analysis indicated no significant differences in the plasma levels of lncRNA BANCR between diabetic patients and healthy controls on the day of admission, indicating that lncRNA BANCR is unlikely involved in the development of diabetes (Fig. 1).

Plasma levels of IncRNA BANCR increase in diabetic patients developing retinopathy but not in diabetic patients developing other or no complications during follow-up. During the 8-year follow-up, continuous decreases were observed in the plasma levels of lncRNA BANCR among patients who developed diabetic retinopathy. However, no significant changes in the plasma levels of lncRNA BANCR were identified among patients who developed diabetic cardiomyopathy, diabetic neuropathy, other complications or no complications (Fig. 2).

Plasma levels of IncRNA BANCR at 12 months prior to diagnosis is able to distinguish patients who will develop diabetic retinopathy from healthy controls and diabetic patients without obvious complications. The diagnostic value of plasma lncRNA BANCR for diabetic retinopathy was 

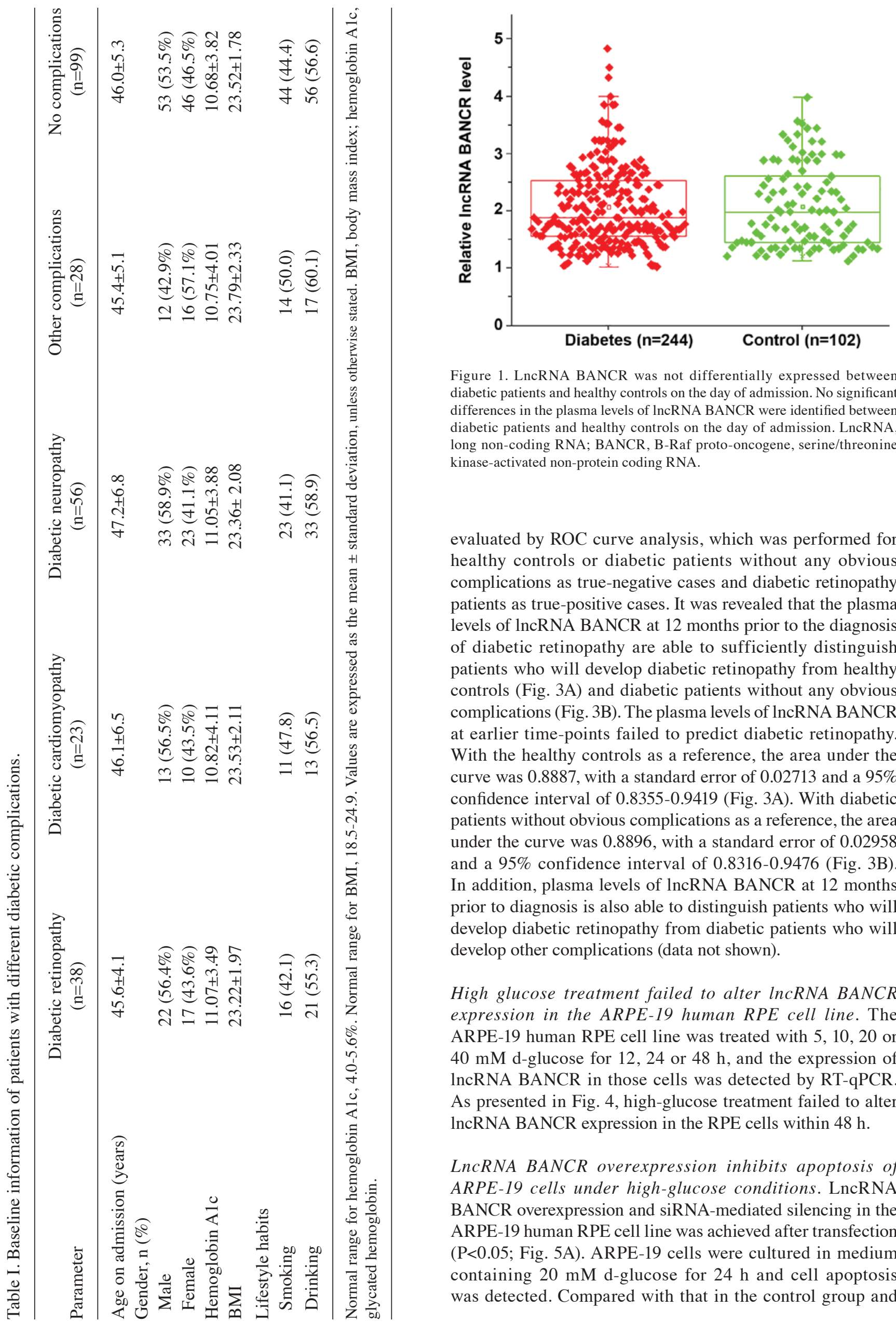

Figure 1. LncRNA BANCR was not differentially expressed between diabetic patients and healthy controls on the day of admission. No significant differences in the plasma levels of IncRNA BANCR were identified between diabetic patients and healthy controls on the day of admission. LncRNA, long non-coding RNA; BANCR, B-Raf proto-oncogene, serine/threonine kinase-activated non-protein coding RNA.

evaluated by ROC curve analysis, which was performed for healthy controls or diabetic patients without any obvious complications as true-negative cases and diabetic retinopathy patients as true-positive cases. It was revealed that the plasma levels of lncRNA BANCR at 12 months prior to the diagnosis of diabetic retinopathy are able to sufficiently distinguish patients who will develop diabetic retinopathy from healthy controls (Fig. 3A) and diabetic patients without any obvious complications (Fig. 3B). The plasma levels of lncRNA BANCR at earlier time-points failed to predict diabetic retinopathy. With the healthy controls as a reference, the area under the curve was 0.8887 , with a standard error of 0.02713 and a $95 \%$ confidence interval of 0.8355-0.9419 (Fig. 3A). With diabetic patients without obvious complications as a reference, the area under the curve was 0.8896 , with a standard error of 0.02958 and a 95\% confidence interval of 0.8316-0.9476 (Fig. 3B). In addition, plasma levels of lncRNA BANCR at 12 months prior to diagnosis is also able to distinguish patients who will develop diabetic retinopathy from diabetic patients who will develop other complications (data not shown).

High glucose treatment failed to alter IncRNA BANCR expression in the ARPE-19 human RPE cell line. The ARPE-19 human RPE cell line was treated with 5, 10, 20 or $40 \mathrm{mM} \mathrm{d}$-glucose for 12,24 or $48 \mathrm{~h}$, and the expression of IncRNA BANCR in those cells was detected by RT-qPCR. As presented in Fig. 4, high-glucose treatment failed to alter lncRNA BANCR expression in the RPE cells within $48 \mathrm{~h}$.

LncRNA BANCR overexpression inhibits apoptosis of ARPE-19 cells under high-glucose conditions. LncRNA BANCR overexpression and siRNA-mediated silencing in the ARPE-19 human RPE cell line was achieved after transfection ( $\mathrm{P}<0.05$; Fig. 5A). ARPE-19 cells were cultured in medium containing $20 \mathrm{mM} \mathrm{d}$-glucose for $24 \mathrm{~h}$ and cell apoptosis was detected. Compared with that in the control group and 


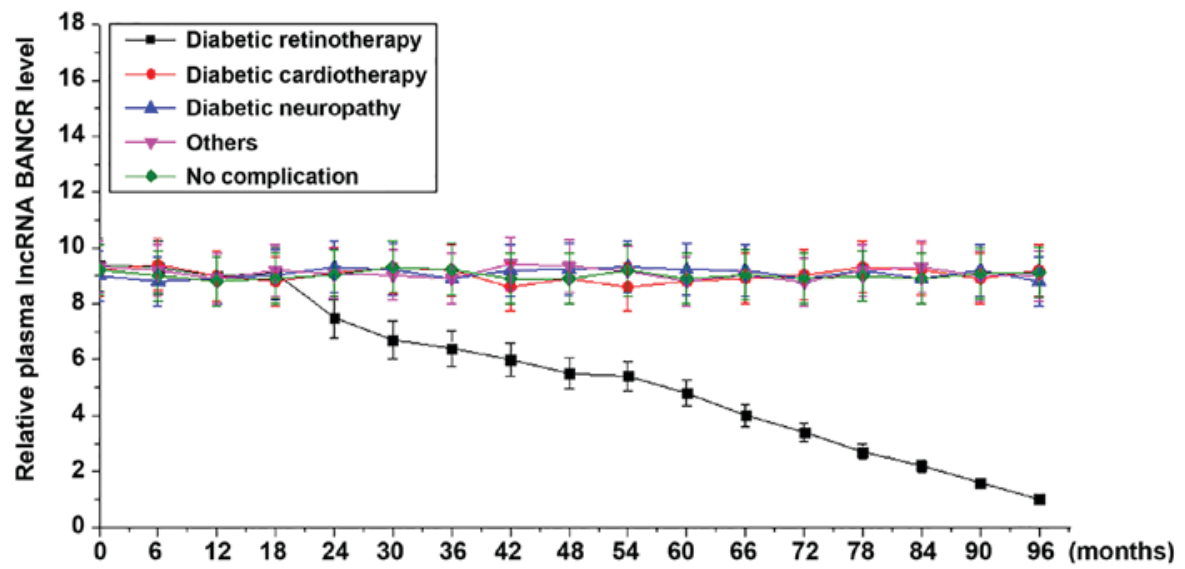

Figure 2. The plasma levels of lncRNA BANCR increased in diabetic retinopathy patients but not in diabetic patients with other or no complications during follow-up. During follow-up, continuous decreases were observed in plasma levels of lncRNA BANCR among patients who developed diabetic retinopathy but not among diabetic patients with other or no complications. LncRNA, long non-coding RNA; BANCR, B-Raf proto-oncogene, serine/threonine kinase-activated non-protein coding RNA.
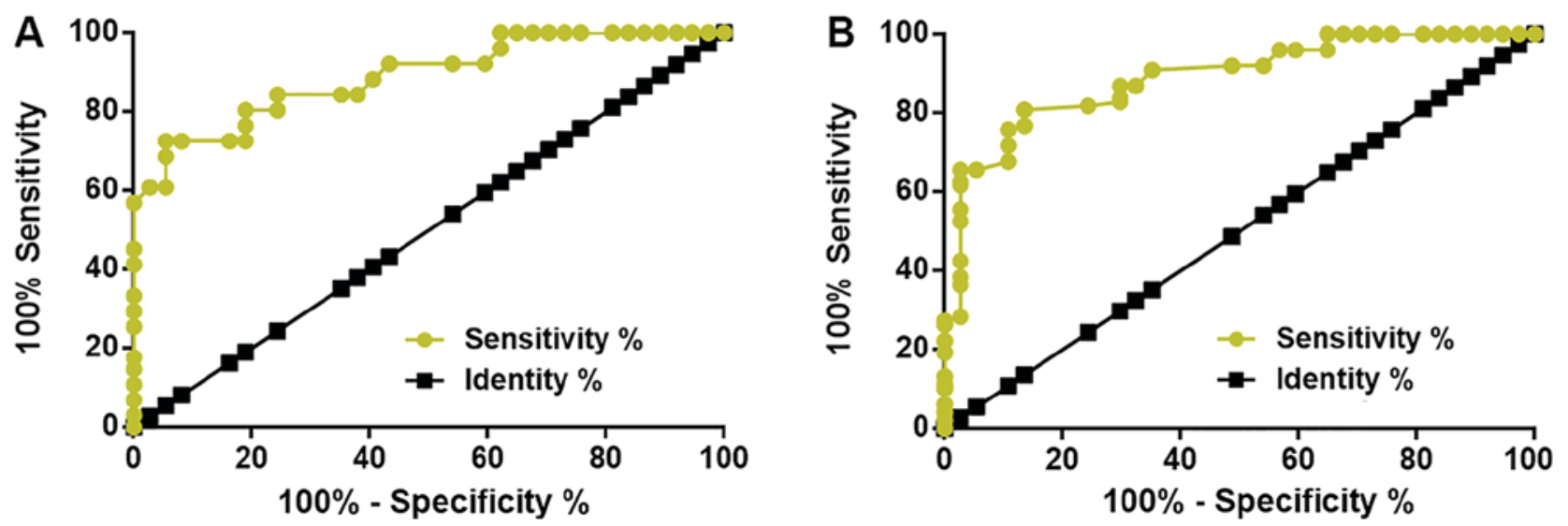

Figure 3. Plasma levels of lncRNA BANCR at 12 months prior to diagnosis are able to distinguish diabetic retinopathy patients from healthy controls and diabetic patients without any obvious complications. LncRNA BANCR at 12 months prior to diagnosis of diabetic retinopathy is able to distinguish diabetic retinopathy patients from (A) healthy controls and (B) diabetic patients without any obvious complications. LncRNA, long non-coding RNA; BANCR, B-Raf proto-oncogene, serine/threonine kinase-activated non-protein coding RNA.

the negative control group, IncRNA BANCR overexpression inhibited, while IncRNA BANCR silencing promoted the apoptosis of ARPE-19 cells ( $\mathrm{P}<0.05$; Fig. 5B).

\section{Discussion}

The functions of IncRNA BANCR have been well characterized in cancer biology $(10,11)$, while its involvement in diabetic complications has remained elusive. The key result of the present study is that IncRNA BANCR is downregulated in diabetic retinopathy and ectopic overexpression of lncRNA inhibits the apoptosis of RPE cells under high-glucose conditions.

Previous studies have indicated that the development of diabetic retinopathy globally affects the expression of lncRNAs, indicating the involvement of lncRNAs in this disease $(16,17)$. However, studies on the involvement of IncRNAs in diabetic retinopathy are rare. In a recent study, Zhang et al (18) reported that lncRNA maternally expressed 3 (MEG3) was downregulated in diabetic retinopathy and overexpression of IncRNA MEG3 may improve the condition



Figure 4. High-glucose treatment failed to alter lncRNA BANCR expression in the ARPE-19 human retinal pigment epithelial cell line. LncRNA, long non-coding RNA; BANCR, B-Raf proto-oncogene, serine/threonine kinase-activated non-protein coding RNA.

of this disease by regulating the expression of transforming growth factor- $\beta 1$ and vascular endothelial growth factor. In 

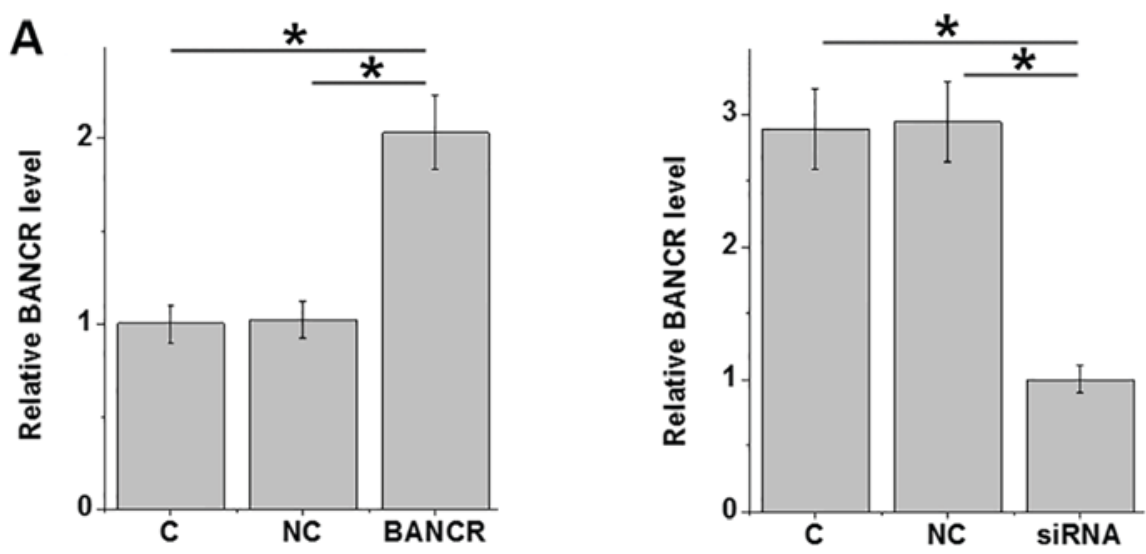

B
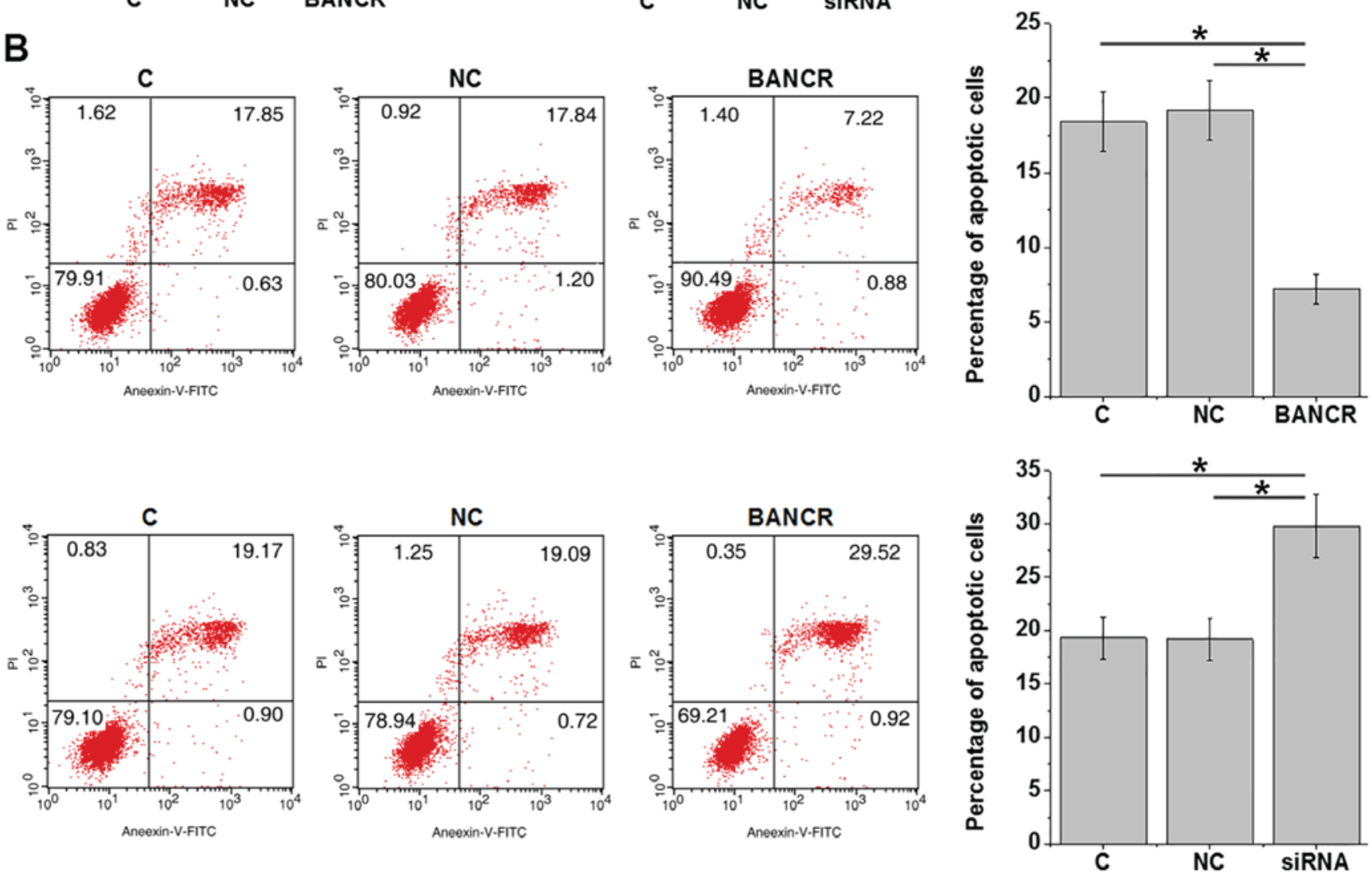

Figure 5. LncRNA BANCR overexpression inhibits apoptosis of cells of ARPE-19 human retinal pigment epithelial cells under high-glucose conditions (A) LncRNA BANCR overexpression and siRNA-mediated silencing was achieved in ARPE-19 cells after transfection. (B) LncRNA BANCR overexpression inhibited, while siRNA silencing promoted the apoptosis of ARPE-19 cells under high-glucose treatment. "P<0.05. LncRNA, long non-coding RNA; BANCR, B-Raf proto-oncogene, serine/threonine kinase-activated non-protein coding RNA; siRNA, small interfering RNA; C, non-transfected control; NC, negative control; PI, propidium iodide; FITC, fluorescein isothiocyanate.

another study, Biswas et al (19) indicated that lncRNA metastasis-associated lung adenocarcinoma transcript 1 and HOX transcript antisense RNA are the key epigenetic regulators in diabetic retinopathy-associated pathological changes. In the present study, it was demonstrated that IncRNA BANCR was downregulated specifically in patients with diabetic retinopathy but not in patients with other diabetic complications. In vitro cell experiments using the ARPE-19 human RPE cell line also suggested that lncRNA BANCR expression was not affected by a high-glucose environment, whereas BANCR overexpression inhibited the apoptosis of ARPE-19 cells under high glucose treatment. The present authors speculate that BANCR may be affected by long-term, but not short-term, high glucose treatment, or BANCR downregulation is caused by the formation of lesions in the eyes. Nevertheless, the present ROC curve analysis data suggested that lncRNA BANCR may be a specific biomarker for diabetic retinopathy due to the observation that altered BANCR distinguished diabetic retinopathy patients from healthy controls, diabetic patients without obvious complications and diabetic patients with other complications.

Blindness inevitably occurs in certain patients with diabetic retinopathy. For the efficient management of diabetic retinopathy, early detection and prediction of risks are critical (20). In the present study, it was demonstrated that the plasma levels of IncRNA BANCR at 12 months prior to the diagnosis of diabetic retinopathy are able to sufficiently distinguish patients who will develop diabetic retinopathy from healthy controls 
and diabetic patients without any obvious complications. Therefore, circulating lncRNA BANCR may be of predictive value for diabetic retinopathy.

Accelerated apoptosis of human RPE cells is a major pathological change in eyes of patients with diabetic retinopathy and inhibition of human RPE cell apoptosis is considered as a promising therapeutic strategy for diabetic retinopathy (21). In the present study, IncRNA BANCR overexpression inhibited the apoptosis of human RPE cells in a high-glucose environment. Therefore, lncRNA BANCR overexpression may be a potential strategy for the treatment of diabetic retinopathy. However, the molecular mechanisms of the regulatory role of lncRNA BANCR in the apoptosis of human RPE cells remain elusive

It is worth noting that the present study failed to detect the expression of IncRNA BANCR in aqueous humor or vitreous specimens. Future studies by our group will aim at performing this analysis. In addition, the present study also failed to explore the molecular mechanisms of BANCR in diabetic retinopathy. Future studies by our group will investigate the molecular mechanisms of the action of BANCR in diabetic retinopathy. However, the present study suggests that BANCR may serve as a potential therapeutic target for diabetic retinopathy, which provides a novel approach for the clinical treatment of this disease. In the present study, only Annexin V-FITC and PI to were used to detect cell apoptosis. However, apoptotic modulation at the molecular level should be validated by further approaches, including the detection of cleavage of caspases and poly(ADP ribose) polymerase and DNA laddering, and the presence of apoptosis should be screened by microscopic view of nuclear condensation. The present study failed to perform these analyses due to limited resources. In future studies, these experiments may be performed to further confirm the present conclusions.

In conclusion, the present study revealed that IncRNA BANCR was downregulated in diabetic retinopathy and that overexpression of IncRNA BANCR to inhibit cell apoptosis may be a novel therapeutic approach to improve diabetic retinopathy.

\section{Acknowledgements}

Not applicable.

\section{Funding}

No funding received.

\section{Availability of data and materials}

All data generated or analyzed during the present study are included in this published article.

\section{Author's contributions}

$\mathrm{XZh}, \mathrm{XZo}, \mathrm{YL}$ and $\mathrm{YW}$ were responsible for the conception and design of the study. XZh, XZo and YL performed the experiments. XZh, XZo and YL analyzed and interpreted the data. XZh and YW drafted the manuscript. XZh, XZo, YL and $\mathrm{YW}$ were responsible for the revision of the manuscript.

\section{Ethics approval and consent to participate}

The protocol of the present study was approved by the Ethics Review Committee of Lanzhou University Second Hospital (Lanzhou, China). All participants provided written informed consent.

\section{Patient consent for publication}

Not applicable.

\section{Competing interests}

The authors declare that they have no competing interests.

\section{References}

1. Semeraro F, Cancarini A, dell'Omo R, Rezzola S, Romano MR and Costagliola C: Diabetic retinopathy: Vascular and inflammatory disease. J Diabetes Res 2015: 582060, 2015.

2. Lee R, Wong TY and Sabanayagam C: Epidemiology of diabetic retinopathy, diabetic macular edema and related vision loss. Eye Vis (Lond) 2: 17, 2015.

3. Ting DS, Cheung GC and Wong TY: Diabetic retinopathy: Global prevalence, major risk factors, screening practices and public health challenges: A review. Clin Exp Ophthalmol 44: 260-277, 2016.

4. Wang C, Li J, Xue H, Li Y, Huang J, Mai J, Chen J, Cao J, Wu X, Guo D, et al: Type 2 diabetes mellitus incidence in Chinese: Contributions of overweight and obesity. Diabetes Res Clin Pract 107: 424-432, 2015.

5. Guariguata L, Whiting DR, Hambleton I, Beagley J, Linnenkamp U and Shaw JE: Global estimates of diabetes prevalence for 2013 and projections for 2035. Diabetes Res Clin Pract 103: 137-149, 2014.

6. Simó R and Hernández C: Novel approaches for treating diabetic retinopathy based on recent pathogenic evidence. Prog Retin Eye Res 48: 160-180, 2015.

7. Leasher JL, Bourne RRA, Flaxman SR, Jonas JB, Keeffe J, Naidoo N, Pesudovs K, Price H, White RA, Wong TY, et al: Global estimates on the number of people blind or visually impaired by diabetic retinopathy: A meta-analysis from 1990 to 2010. Diabetes Care 39: 1643-1649, 2016.

8. Shi X, Sun M, Liu H, Yao Y and Song Y: Long non-coding RNAs: A new frontier in the study of human diseases. Cancer Lett 339: 159-166, 2013.

9. Esteller M: Non-coding RNAs in human disease. Nat Rev Genet 12: 861-874, 2011.

10. Li L, Zhang L, Zhang Y and Zhou F: Increased expression of LncRNA BANCR is associated with clinical progression and poor prognosis in gastric cancer. Biomed Pharmacother 72: 109-112, 2015.

11. Wang D, Wang D, Wang N, Long Z and Ren X: Long non-coding RNA BANCR promotes endometrial cancer cell proliferation and invasion by regulating MMP2 and MMP1 via ERK/MAPK signaling pathway. Cell Physiol Biochem 40: 644-656, 2016.

12. Su S, Gao J, Wang T, Wang J, Li H and Wang Z: Long non-coding RNA BANCR regulates growth and metastasis and is associated with poor prognosis in retinoblastoma. Tumuor Biol 36: 7205-7211, 2015.

13. Dunn KC, Aotaki-Keen AE, Putkey FR and Hjelmeland LM: ARPE-19, a human retinal pigment epithelial cell line with differentiated properties. Exp Eye Res 62: 155-170, 1996.

14. Livak KJ and Schmittgen TD: Analysis of relative gene expression data using real-time quantitative PCR and the 2(-Delta Delta C(T)) method. Methods 25: 402-408, 2001.

15. Cregan SP, Smith BP, Brown DL and Mitchel RE: Two pathways for the induction of apoptosis in human lymphocytes. Int J Radiat Biol 75: 1069-1086, 1999.

16. Yan B, Tao ZF, Li XM, Zhang H, Yao J and Jiang Q: Aberrant expression of long noncoding RNAs in early diabetic retinopathy. Invest Ophthalmol Vis Sci 55: 941-951, 2014.

17. Jaé $\mathrm{N}$ and Dimmeler S: Long noncoding RNAs in diabetic retinopathy. Circ Res 116: 1104-1106, 2015. 
18. Zhang D, Qin H, Leng Y, Li X, Zhang L, Bai D, Meng Y and Wang J: LncRNA MEG3 overexpression inhibits the development of diabetic retinopathy by regulating TGF- $\beta 1$ and VEGF. Exp Ther Med 16: 2337-2342, 2018.

19. Biswas S, Thomas AA, Feng B, Chen S, Aref-Eshghi E, Gonder J, Sadikovic B and Chakrabarti S: MALAT1 and HOTAIR-Key epigenetic regulators in diabetic retinopathy. Diabetes 67: 240, 2018

20. Olafsdottir E, Andersson DKG, Dedorsson I, Svärdsudd K, Jansson SP and Stefánsson E: Early detection of type 2 diabetes mellitus and screening for retinopathy are associated with reduced prevalence and severity of retinopathy. Acta Ophthalmol 94: 232-239, 2016.
21. Kim DI, Park MJ, Lim SK, Choi JH, Kim JC, Han HJ, Kundu TK, Park JI, Yoon KC, Park SW, et al: High-glucose-induced CARM1 expression regulates apoptosis of human retinal pigment epithelial cells via histone 3 arginine 17 dimethylation: Role in diabetic retinopathy. Arch Biochem Biophys 560: 36-43, 2014.

This work is licensed under a Creative Commons Attribution-NonCommercial-NoDerivatives 4.0 International (CC BY-NC-ND 4.0) License. 